\title{
Inference Based on Type-II Hybrid Censored Data From a Weibull Distribution
}

\author{
Aveek Banerjee and Debasis Kundu
}

\begin{abstract}
A hybrid censoring scheme is a mixture of Type-I and Type-II censoring schemes. This article presents the statistical inferences on Weibull parameters when the data are Type-II hybrid censored. The maximum likelihood estimators, and the approximate maximum likelihood estimators are developed for estimating the unknown parameters. Asymptotic distributions of the maximum likelihood estimators are used to construct approximate confidence intervals. Bayes estimates, and the corresponding highest posterior density credible intervals of the unknown parameters, are obtained using suitable priors on the unknown parameters, and by using Markov Chain Monte Carlo techniques. The method of obtaining the optimum censoring scheme based on the maximum information measure is also developed. We perform Monte Carlo simulations to compare the performances of the different methods, and we analyse one data set for illustrative purposes.
\end{abstract}

Index Terms-Approximate maximum likelihood estimators, asymptotic distribution, Bayes estimators, hybrid censoring, Markov chain Monte Carlo, maximum likelihood estimators, optimum censoring scheme, Type-I censoring, Type-II censoring.

\section{ACRONYM $^{1}$}

MLE maximum likelihood estimator

AMLE approximate maximum likelihood estimator

CDF cumulative distribution function

PDF probability density function

K-S Kolmogorov-Smirnov

EM expectation maximization

w.r.t with respect to

r.h.s. right hand side

HPD highest posterior density

MCMC Markov chain Monte Carlo

MSE mean squared error

BEL Bayes estimates using Lindley's approximation

BEM Bayes estimates using MCMC

\section{NOTATION}

$Y_{i: n} \quad i$-th ordered failure time when the sample size is $n$

Manuscript received February 2, 2007; revised June 14, 2007 and August 3, 2007; accepted August 5, 2007. Associate Editor: M. Xie.

A. Banerjee was with the Indian Institute of Technology Kanpur, Kanpur 208016, India. He is now with Accenture, Bangalore, India.

D. Kundu is with the Department of Mathematics and Statistics, Indian Institute of Technology Kanpur, Kanpur 208016, India.

Digital Object Identifier 10.1109/TR.2008.916890

${ }^{1}$ The singular and plural of an acronym are always spelled the same.

\author{
Weibull $(\alpha, \lambda)$ Weibull random variable with PDF \\ $\alpha \lambda x^{\alpha-1} e^{-\lambda x^{\alpha}}$ \\ $\operatorname{Gamma}(\alpha, \lambda)$ gamma random variable with PDF \\ $\lambda^{\alpha} /(\Gamma(\alpha)) x^{\alpha-1} e^{-\lambda x}$ \\ $E V(\mu, \sigma) \quad$ extreme value random variable with PDF \\ $(1 / \sigma) e^{((x-\mu) / \sigma)-e^{((x-\mu) / \sigma)}}$ \\ $[T, R] \quad$ Type-I hybrid censoring scheme \\ $(T, R) \quad$ Type-II hybrid censoring scheme
}

\section{INTRODUCTION}

$A$ HYBRID censoring scheme is a mixture of Type-I and Type-II censoring schemes, and it can be described as follows. Suppose $n$ identical units are put to test. The test is terminated when a pre-chosen number $R$ out of $n$ items are failed, or when a pre-determined time $T$ on the test has been reached. It is also assumed that the failed items are not replaced. From now on, we call this the Type-I hybrid censoring scheme. Epstein [11] introduced this Type-I hybrid censoring scheme, and considered lifetime experiments assuming that the lifetime of each unit follows an exponential distribution. He obtained the estimate, and proposed two-sided confidence interval of the unknown parameter without any formal proof. The Type-I hybrid censoring scheme has been used as a reliability acceptance test in MIL-STD-781 C [21]. Since then, several articles have been published on Type-I hybrid censoring schemes; see for example Fairbanks \& Madison \& Dykstra [12], Draper \& Guttman [8], Chen \& Bhattacharya [5], Ebrahimi [9], [10], Jeong \& Park \& Yum [16], Gupta \& Kundu [14], Childs et al. [6], and Kundu [18].

Like the conventional Type-I censoring scheme, the disadvantage of the Type-I hybrid censoring scheme is that all the inferential results are obtained under the condition that the number of observed failures is at least one, and in addition there may be very few failures occurring up to the pre-fixed time $T$. Because of that, Childs et al. [6] proposed a new hybrid censoring scheme known as the Type-II hybrid censoring scheme, and it can be described as follows. Put $n$ identical items on test, and then stop the experiment at the random time $T^{*}=\max \left\{Y_{R: n}, T\right\}$, where $R$, and $T$ are prefixed numbers. Thus, the Type-II hybrid censoring scheme ensures that at least $R$ failures take place. Childs et al. [6] mentioned that Such a censoring scheme may arise in a situation when the experimenter determines that at least $r$ failures must be observed and has prepaid for the use of the testing facility for $T$ units of time. If the $r$ failures occur before time $T$, then the experiment can continue up to time $T$ to make full use of the testing facility. If the r-th failure does not 

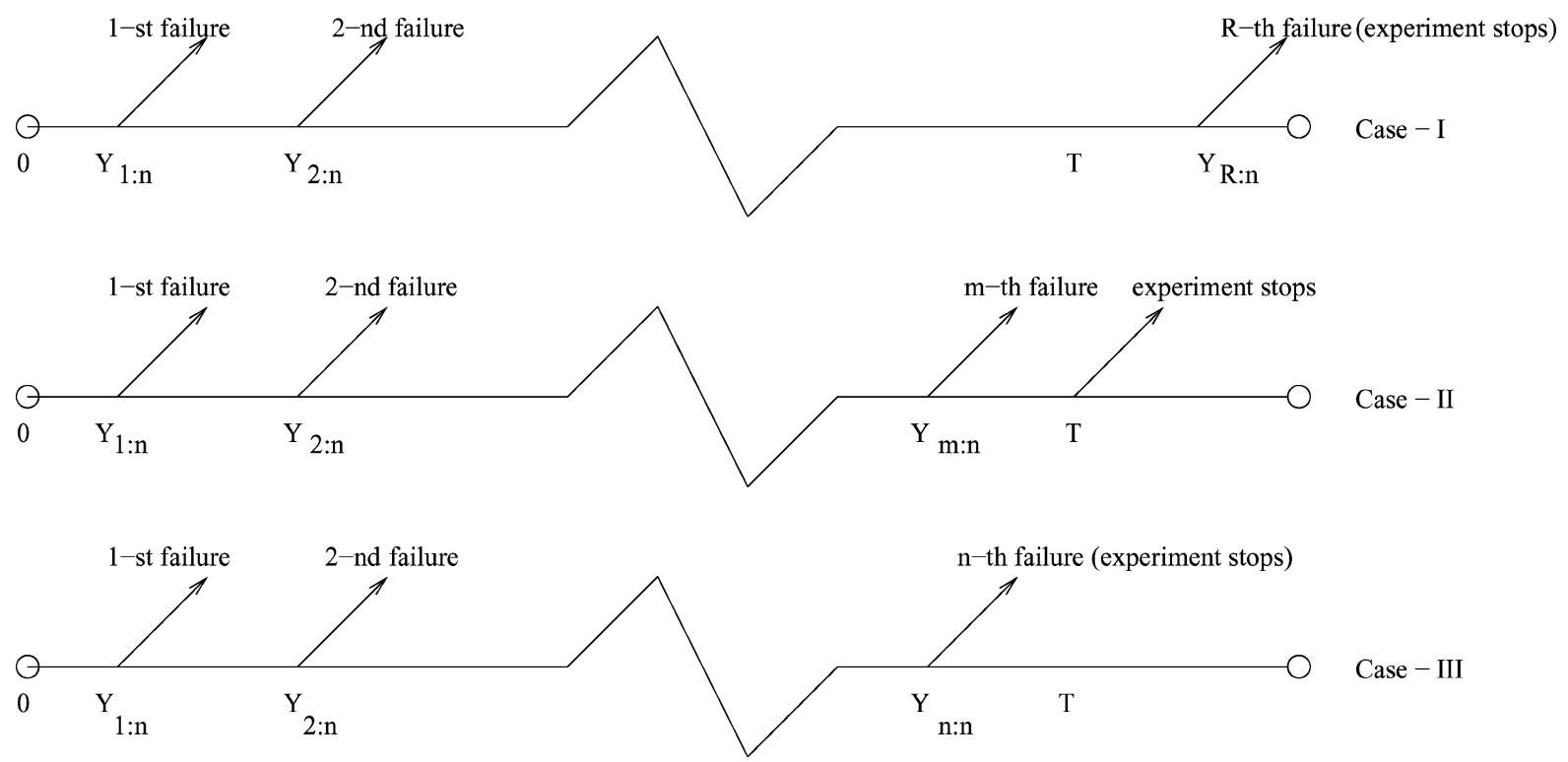

Fig. 1. Schematic illustration of the Type-II hybrid censoring scheme.

occur before time $T$ then he/she will naturally choose to continue until $r$-th failure. For the comparison of the two hybrid censoring schemes, the readers are referred to Childs et al. [6].

Because the lifetime distributions of many experimental units can be modeled by a two-parameter Weibull distribution, in this paper we consider Type-II hybrid censoring lifetime data when the lifetime distribution of each unit follows a two-parameter Weibull distribution. We also assume that the failed items are not replaced. First, we provide the MLE of the unknown parameters, and it is observed that the MLE can not be obtained in explicit forms. The MLE of the two unknown parameters can be obtained by solving one non-linear equation. We provide a simple fixed point type algorithm to find the MLE. We also provide the AMLE which can be obtained in explicit forms. Because the exact distributions of the MLE are not easy to derive, we propose to use the asymptotic distributions of the MLE to construct the approximate confidence intervals.

We also consider the Bayesian solution of this problem. For the Weibull distribution, no natural continuous conjugate priors exist simultaneously for both the shape, and scale parameters; see for example Kaminskiy \& Krivtsov [17]. When the shape parameter is known, the gamma prior is the natural conjugate prior on the scale parameter. So we assume the gamma prior on the scale parameter. For the shape parameter also, we have considered the gamma prior. Based on these priors, the Bayes estimates can not be obtained explicitly. Because the Bayes estimates can not be obtained explicitly, we provide two approximations, namely Lindley's approximation, and the Gibbs sampling procedure. We use the Gibbs sampling procedure to compute the Bayes estimates, and the HPD credible intervals. We compare the performances of the different methods by Monte Carlo simulations, and for illustrative purposes we have analysed one real data set.

The rest of the paper is organize as follows. In Section II, we describe the model, and the available data. The MLE, and AMLE are presented in Sections III and IV respectively.
Bayesian analyses are presented in Section V. Simulation results, and data analysis are provided in Sections VI and VII respectively. We provide a simple heuristic procedure to find the optimum censoring scheme in Section VIII, and finally we conclude the paper in Section IX.

\section{MODEL, AND DATA DESCRIPTIONS}

Suppose the lifetime $Y$ has a $W$ eibull $(\alpha, \lambda)$. Now consider the transformed random variable $X=\ln Y$. If $Y$ has a $W$ eibull $(\alpha, \lambda)$, then $X$ has $E V(\mu, \sigma)$, where $\sigma=1 / \alpha$, and $\mu=-\ln \lambda / \alpha$. Here, $\mu$, and $\sigma$ are the location, and scale parameters respectively.

It is well known that $W$ eibull $(\alpha, \lambda)$, and $E V(\mu, \sigma)$ are equivalent models. Because $E V(\mu, \sigma)$ belongs to the location-scale family, sometimes it is easier to work with $E V(\mu, \sigma)$ rather than $W$ eibull $(\alpha, \lambda)$. In $E V(\mu, \sigma)$, if we put $\mu=0$, and $\sigma=1$, then it reduces to the standard extreme value distribution.

Now we describe the data available under the Type-II hybrid censoring scheme. For known $R$, and $T$, we can observe the following three types of observations.

CASE I: $\left\{y_{1: n}<\ldots<y_{R: n}\right\}$ if $y_{R: n}>T$.

CASE II: $\left\{y_{1: n}<\ldots<y_{R: n}<y_{R+1: n}<\ldots<y_{m: n}<\right.$ $\left.T<y_{m+1: n}\right\}$, if $R \leq m<n$, and $y_{m: n}<T<y_{m+1: n}$. CASE III: $\left\{y_{1: n}<\ldots<y_{n: n}<T\right\}$.

Note that, in case II, we do not observe $y_{m+1: n}$, but $y_{m: n}<$ $T<y_{m+1: n}$ means that the $m$-th failure took place before $T$, and no failure took place between $y_{m: n}$ and $T$. A schematic representation of the Type-II hybrid censoring scheme is presented in Fig. 1. In the next section, we obtain the MLE of $\alpha$, and $\lambda$ based on the observed sample.

\section{MAXIMUM LIKELIHOOD ESTIMATORS}

In this section, we provide the MLE of $\alpha$, and $\lambda$ for three different cases. The likelihood functions for three different cases follow. 


\section{CASE I}

$$
l(\alpha, \lambda)=\alpha^{R} \lambda^{R} \prod_{i=1}^{R} y_{i: n}^{\alpha-1} e^{-\lambda\left[\sum_{i=1}^{R} y_{i: n}^{\alpha}+(n-R) y_{R: n}^{\alpha}\right]}
$$

\section{CASE II}

$$
l(\alpha, \lambda)=\alpha^{m} \lambda^{m} \prod_{i=1}^{m} y_{i: n}^{\alpha-1} e^{-\lambda\left[\sum_{i=1}^{m} y_{i: n}^{\alpha}+(n-m) T^{\alpha}\right]}
$$

CASE III

$$
l(\alpha, \lambda)=\alpha^{n} \lambda^{n} \prod_{i=1}^{n} y_{i: n}^{\alpha-1} e^{-\lambda \sum_{i=1}^{n} y_{i: n}^{\alpha}} .
$$

Therefore, Cases I, II, and III can be combined, and can be written as

$$
l(\alpha, \lambda)=\alpha^{D} \lambda^{D} \prod_{i=1}^{D} y_{i: n}^{\alpha-1} e^{-\lambda\left[\sum_{i=1}^{D} y_{i: n}^{\alpha}+(n-D) U^{\alpha}\right]} .
$$

Here, $D$ denotes the number of failures; and $U=y_{R: n}$ if $D=$ $R$, and $U=T$ if $D>R$. From (4), it is clear that the MLE of $\alpha$, and $\lambda$ can be obtained by solving the following two equations.

$$
\begin{aligned}
& \frac{D}{\lambda}-\left[\sum_{i=1}^{D} y_{i: n}^{\alpha}+(n-D) y_{D: n}^{\alpha}\right]=0, \\
& \frac{D}{\alpha}+\sum_{i=1}^{D} \ln y_{i: n} \\
& \quad-\lambda\left[\sum_{i=1}^{D} y_{i: n}^{\alpha} \ln y_{i: n}+(n-D) U^{\alpha} \ln U\right]=0 .
\end{aligned}
$$

Therefore, for fixed $\alpha$, the MLE of $\lambda$, say $\widehat{\lambda}(\alpha)$, can be obtained as

$$
\widehat{\lambda}(\alpha)=\frac{D}{\sum_{i=1}^{D} y_{i: n}^{\alpha}+(n-D) U^{\alpha}},
$$

and the MLE of $\alpha$ can be obtained by solving the non-linear equation

$\frac{D}{\alpha}+\sum_{i=1}^{D} \ln y_{i: n}-\widehat{\lambda}(\alpha)\left[\sum_{i=1}^{D} y_{i: n}^{\alpha} \ln y_{i: n}+(n-D) U^{\alpha} \ln U\right]=0$.

Equation (8) can be written as

$$
h(\alpha)=\alpha,
$$

where

$$
h(\alpha)=\frac{D}{\widehat{\lambda}(\alpha)\left[\sum_{i=1}^{D} y_{i: n}^{\alpha} \ln y_{i: n}+(n-D) U^{\alpha} \ln U\right]-\sum_{i=1}^{D} \ln y_{i: n}} .
$$

We propose a simple iterative procedure to solve for (9). Start with some initial guess of $\alpha$, say $\alpha^{(0)}$, then compute $\alpha^{(1)}=$ $h\left(\alpha^{(0)}\right)$. Proceed in this way to obtain $\alpha^{(n+1)}=h\left(\alpha^{(n)}\right)$. Stop the iterative procedure when $\left|\alpha^{(n+1)}-\alpha^{(n)}\right|<\epsilon$, some preassigned tolerance limit. Once we obtain the MLE of $\alpha$, say $\widehat{\alpha}$, then the MLE of $\lambda$ can be obtained as $\widehat{\lambda}=\widehat{\lambda}(\widehat{\alpha})$ from (7).

\section{APPROXIMATE MAXIMUM LIKELIHOOD ESTIMATORS}

Because the MLE can not be obtained in explicit forms, in this section we propose the AMLE which have explicit forms. Let $x_{i: n}=\ln y_{i: n}$. Therefore, the likelihood equation of the observed data can be written as

$$
l(\mu, \sigma) \propto \frac{1}{\sigma^{D}} \prod_{i=1}^{D} g\left(z_{i: n}\right)(\bar{G}(V))^{n-D},
$$

where

$$
\begin{gathered}
g(x)=e^{x-e^{x}}, \quad \bar{G}(x)=e^{-e^{x}}, \quad z_{i: n}=\frac{x_{i: n}-\mu}{\sigma}, \\
V=\frac{U-\mu}{\sigma}, \quad \sigma=\frac{1}{\alpha}, \quad \mu=-\frac{1}{\alpha} \ln \lambda .
\end{gathered}
$$

Ignoring the constant, and taking the logarithm of (11), we obtain

$$
L(\mu, \sigma)=-D \ln \sigma+\sum_{i=1}^{D} \ln g\left(z_{i: n}\right)+(n-D) \ln (\bar{G}(V)) .
$$

Taking derivatives of $L(\mu, \sigma)$, with respect to $\mu$, and $\sigma$, gives

$$
\begin{aligned}
\frac{\partial L}{\partial \mu}= & -\frac{1}{\sigma} \sum_{i=1}^{D} \frac{g^{\prime}\left(z_{i: n}\right)}{g\left(z_{i: n}\right)}+\frac{1}{\sigma}(n-D) \frac{g(V)}{\bar{G}(V)}=0, \\
\frac{\partial L}{\partial \sigma}= & -\frac{D}{\sigma}-\frac{1}{\sigma} \sum_{i=1}^{D} \frac{g^{\prime}\left(z_{i: n}\right)}{g\left(z_{i: n}\right)} z_{i: n} \\
& +\frac{1}{\sigma}(n-D) \frac{g(V)}{\bar{G}(V)} V=0 .
\end{aligned}
$$

Clearly, (13), and (14) do not have explicit solutions. We expand the functions $g^{\prime}\left(z_{i: n}\right) / g\left(z_{i: n}\right)$, and $g(V) / \bar{G}(V)$ in Taylor series as follows. Suppose $p_{i}=i /(n+1), q_{i}=1-p_{i}$ for $i=1, \ldots, n$, and $p_{D^{*}}=\left(p_{D}+p_{D+1}\right) / 2, q_{D^{*}}=1-p_{D^{*}}$ for $1 \leq D \leq$ $n-1$. Then, expand the function $g^{\prime}\left(z_{i: n}\right) / g\left(z_{i: n}\right)$ around the point $G^{-1}\left(p_{i}\right)=\ln \left(-\ln q_{i}\right)=\mu_{i}$ (say) for $i=1, \ldots, D$. Also, expand the function $g(V) / \bar{G}(V)$ around the point $G^{-1}\left(p_{R}\right)=$ $\mu_{R}$ if $U=y_{R: n}$. If $U=T$, expand $g(V) / \bar{G}(V)$ around the point $G^{-1}\left(p_{D^{*}}\right)=\ln \left(-\ln \left(1-p_{D}^{*}\right)\right)=\mu_{D^{*}}$ (say), when $D<$ $n$. Similar methods were used by Balasooriya \& Balakrishnan [3], and also see Arnold et al. [1]. Note that for $i=1, \ldots, D$,

$$
\frac{g^{\prime}\left(z_{i: n}\right)}{g\left(z_{i: n}\right)} \approx \alpha_{i}-\beta_{i} z_{i: n}
$$

where

$$
\begin{aligned}
\alpha_{i} & =\frac{g^{\prime}\left(\mu_{i}\right)}{g\left(\mu_{i}\right)}-\mu_{i}\left[\frac{g^{\prime \prime}\left(\mu_{i}\right)}{g\left(\mu_{i}\right)}-\left(\frac{g^{\prime}\left(\mu_{i}\right)}{g\left(\mu_{i}\right)}\right)^{2}\right] \\
& =1+\ln q_{i}\left(1-\ln \left(-\ln q_{i}\right)\right) \\
\beta_{i} & =\left[\left(\frac{g^{\prime}\left(\mu_{i}\right)}{g\left(\mu_{i}\right)}\right)^{2}-\frac{g^{\prime \prime}\left(\mu_{i}\right)}{g\left(\mu_{i}\right)}\right]=-\ln q_{i} .
\end{aligned}
$$

If $U=y_{R: n}$, expanding $g(V) / \bar{G}(V)$ around the point $\mu_{R}$, we obtain

$$
\frac{g(V)}{\bar{G}(V)} \approx 1-\alpha_{D}+\beta_{D} z_{D: n} .
$$


Using the approximations (15), and (18) in (13), and (14), we obtain

$$
\begin{aligned}
& -\sum_{i=1}^{D}\left(\alpha_{i}-\beta_{i} z_{i: n}\right)+(n-D)\left(1-\alpha_{D}+\beta_{D} z_{D: n}\right)=0, \\
& -D-\sum_{i=1}^{D}\left(\alpha_{i}-\beta_{i} z_{i: n} z_{i: n}+(n-D)\right. \\
& \quad \times\left(1-\alpha_{D}+\beta_{D} z_{D: n}\right) z_{D: n}=0 .
\end{aligned}
$$

From (19), we obtain solution of $\widehat{\mu}$ as

$$
\widehat{\mu}=A_{i}-B_{I} \widehat{\sigma},
$$

where

$$
\begin{aligned}
A_{I} & =\frac{\sum_{i=1}^{D} \beta_{i} x_{i: n}+\beta_{D}(n-D) x_{D: n}}{\sum_{i=1}^{D} \beta_{i}+\beta_{D}(n-D)}, \\
B_{I} & =\frac{\sum_{i=1}^{D} \alpha_{i}-(n-D)\left(1-\alpha_{D}\right)}{\sum_{i=1}^{D} \beta_{i}+\beta_{D}(n-D)} .
\end{aligned}
$$

From (20), we obtain $\widehat{\sigma}$ as a solution of the quadratic equation

$$
E_{I} \sigma^{2}+F_{I} \sigma-G_{I}=0
$$

where

$$
\begin{aligned}
E_{I}= & D+B_{I} \sum_{i=1}^{D} \alpha_{i}-B_{I}(n-D)\left(1-\alpha_{D}\right) \\
& -B_{I}^{2} \sum_{i=1}^{D} \beta_{i}-B_{I}^{2}(n-D) \beta_{D}=D, \\
F_{I}= & \sum_{i=1}^{D} \alpha_{i}\left(x_{i: n}-A_{I}\right)-(n-D)\left(1-\alpha_{D}\right)\left(x_{D: n}-A_{i}\right) \\
& -2 B_{I} \sum_{i=1}^{D} \beta_{i}\left(x_{i: n}-A_{I}\right)-2(n-D) \beta_{D} B_{I}\left(x_{D: n}-A_{I}\right),
\end{aligned}
$$

and

$$
G_{I}=\sum_{i=1}^{D} \beta_{i}\left(x_{i: n}-A_{I}\right)^{2}+(n-D) \beta_{D}\left(x_{D: n}-A_{I}\right)^{2}>0 .
$$

Therefore,

$$
\widehat{\sigma}=\frac{-F_{I}+\sqrt{F_{I}^{2}+4 D G_{I}}}{2 D},
$$

is the only positive root. If $U=T$, expanding $g(V) / \bar{G}(V)$ around the point $\mu_{D^{*}}$, and following the same procedure as above, we obtain the solution of $\widehat{\mu}$ as

$$
\widehat{\mu}=A_{I I}-B_{I I} \widehat{\sigma},
$$

where

$$
A_{I I}=\frac{\sum_{i=1}^{D} \beta_{i} x_{i: n}+\beta_{D^{*}}(n-D) \ln T}{\sum_{i=1}^{D} \beta_{i}+\beta_{D^{*}}(n-D)}, \quad \text { and }
$$

$$
B_{I I}=\frac{\sum_{i=1}^{D} \alpha_{i}-(n-D)\left(1-\alpha_{D^{*}}\right)}{\sum_{i=1}^{D} \beta_{i}+\beta_{D^{*}}(n-D)} .
$$

Here, $\beta_{D^{*}}=-\ln q_{D^{*}}$, and $\alpha_{D^{*}}=1+\ln q_{D^{*}}(1-$ $\left.\ln \left(-\ln q_{D^{*}}\right)\right)$. Moreover, $\widehat{\sigma}$ can be obtained as

$$
\widehat{\sigma}=\frac{-F_{I I}+\sqrt{F_{I I}^{2}+4 D G_{I I}}}{2 D},
$$

where

$$
\begin{aligned}
F_{I I} & =\sum_{i=1}^{D} \alpha_{i}\left(x_{i: n}-A_{I I}\right)-(n-D)\left(1-\alpha_{D^{*}}\right)\left(\ln T-A_{I I}\right) \\
& -2 B \sum_{i=1}^{D} \beta_{i}\left(x_{i: n}-A_{I I}\right)-2(n-D) \beta_{D^{*}} B\left(\ln T-A_{I I}\right),
\end{aligned}
$$

and

$G_{I I}=\sum_{i=1}^{D} \beta_{i}\left(x_{i: n}-A_{I I}\right)^{2}+(n-D) \beta_{D^{*}}\left(\ln T-A_{I I}\right)^{2}>0$.

\section{BAyes Estimators, AND CREDIBLE INTERVALS}

In this section, we consider the Bayesian solution of the problem. We need to assume some prior distributions of the unknown parameters for the Bayesian inference. Following the approach of Berger \& Sun [4], we assume that $\lambda$ has a $\operatorname{Gamma}(a, b)$ prior with $a>0$, and $b>0$. At this moment, we do not assume any specific form of the prior of $\alpha$, say $\pi_{2}(\alpha)$. We simply assumed that $\pi_{2}(\alpha)$ has the support on $(0, \infty)$, and it is independent of the prior of $\lambda$. Based on the above priors, the joint density of the data, $\alpha$, and $\lambda$, can be written for all three cases as follows.

$$
\begin{aligned}
l(\alpha, \lambda, \text { data }) \propto & \pi_{2}(\alpha) \alpha^{D} \lambda^{D+a-1} \\
& \times \prod_{i=1}^{D} y_{i: n}^{\alpha-1} e^{-\lambda\left(\sum_{i=1}^{D} y_{i: n}^{\alpha}+(n-D) U^{\alpha}+b\right)}
\end{aligned}
$$

The posterior density function of $\alpha$, and $\lambda$, given the data is

$$
l(\alpha, \lambda \mid \text { data }) \propto \frac{l(\alpha, \lambda, \text { data })}{\int_{0}^{\infty} \int_{0}^{\infty} l(\alpha, \lambda, \text { data }) d \alpha d \lambda} .
$$

Therefore, if $g(\alpha, \lambda)$ is any function of $\alpha$, and $\lambda$, then the Bayes estimate of $g(\alpha, \lambda)$ under a squared error loss is

$$
\widehat{g_{B}}(\alpha, \lambda)=\frac{\int_{0}^{\infty} g(\alpha, \lambda) l(\alpha, \lambda, d a t a) d \alpha d \lambda}{\int_{0}^{\infty} \int_{0}^{\infty} l(\alpha, \lambda, d a t a) d \alpha d \lambda} .
$$

Unfortunately, we can not compute explicitly (30) in most cases; therefore, we adopt two different procedures to approximate (30): (a) Lindley's approximation, and (b) Gibbs sampling procedure.

\section{A. Lindley's Approximation}

In this section, we specify the prior on $\alpha$ to calculate the approximate Bayes estimates of $\alpha$, and $\lambda$. It is already assumed 
that $\lambda$ has the prior $\operatorname{Gamma}(a, b)$, and it is assumed that $\pi_{2}(\alpha)$ also follows $\operatorname{Gamma}(c, d)$. Now, based on the above priors, we compute the approximate Bayes estimates of $\alpha$ and $\lambda$ using Lindley's approximations. Lindley [20] first proposed his procedure to approximate the ratio of two integrals such as (30). Several others used this approximation; see Press [22] for details. Based on Lindley's approximation, the approximate Bayes estimates of $\alpha$, and $\lambda$ for the squared error loss functions are respectively

$$
\begin{aligned}
\widehat{\alpha}_{B}=\widehat{\alpha}+\frac{1}{2}[ & \left\{\frac{2 D}{\widehat{\alpha}^{3}}-\widehat{\lambda}\left(\sum_{i=1}^{D} y_{i: n}^{\widehat{\alpha}}\left(\ln y_{i: n}\right)^{3}\right.\right. \\
& \left.\left.+(n-D) U^{\widehat{\alpha}}(\ln U)^{3}\right)\right\} \tau_{11}^{2} \\
& +\frac{2 D}{\widehat{\lambda}^{3}} \tau_{21} \tau_{22}-3 \tau_{11} \tau_{12} \\
& \left.\times\left(\sum_{i=1}^{D} y_{i: n}^{\widehat{\alpha}}\left(\ln y_{i: n}\right)^{2}+(n-D) U^{\widehat{\alpha}}(\ln U)^{2}\right)\right] \\
+ & \tau_{11}\left(\frac{c-1}{\widehat{\alpha}}-d\right)+\tau_{12}\left(\frac{a-1}{\widehat{\lambda}}-b\right),
\end{aligned}
$$

and

$$
\begin{aligned}
\widehat{\lambda}_{B}=\widehat{\lambda}+\frac{1}{2}[ & \left\{\frac{2 D}{\widehat{\alpha}^{3}}-\widehat{\lambda}\left(\sum_{i=1}^{D} y_{i: n}^{\widehat{\alpha}}\left(\ln y_{i: n}\right)^{3}\right.\right. \\
& \left.\left.+(n-D) U^{\widehat{\alpha}}(\ln U)^{3}\right)\right\} \tau_{11} \tau_{12} \\
& +\frac{2 D}{\widehat{\lambda}^{3}} \tau_{22}^{2}-\left(\tau_{11} \tau_{22}+2 \tau_{12}^{2}\right) \\
& \left.\times\left(\sum_{i=1}^{D} y_{i: n}^{\widehat{\alpha}}\left(\ln y_{i: n}\right)^{2}+(n-D) U^{\alpha}(\ln U)^{2}\right)\right] \\
+ & \tau_{21}\left(\frac{c-1}{\widehat{\alpha}}-d\right)+\tau_{22}\left(\frac{a-1}{\widehat{\lambda}}-b\right),
\end{aligned}
$$

where

$$
\tau_{11}=\frac{W}{S W-V^{2}}, \quad \tau_{12}=\tau_{21}=-\frac{V}{S W-V^{2}}, \quad \tau_{22}=\frac{S}{S W-V^{2}},
$$

and

$$
\begin{aligned}
& S=\frac{D}{\widehat{\alpha}^{2}}+\widehat{\lambda}\left[\sum_{i=1}^{D} y_{i: n}^{\widehat{\alpha}}\left(\ln y_{i: n}\right)^{2}+(n-D) U^{\widehat{\alpha}}(\ln U)^{2}\right] \\
& V=\sum_{i=1}^{D} y_{i: n}^{\widehat{\alpha}} \ln y_{i: n}+(n-D) U^{\widehat{\alpha}} \ln U, \quad W=\frac{D}{\widehat{\lambda}^{2}} .
\end{aligned}
$$

For the detailed derivations, see the Appendix, part A.

We can compute the approximate Bayes estimates of $\alpha$ and $\lambda$ using Lindley's approximation, but it is not possible to construct highest posterior density (HPD) credible intervals using this method. Therefore, we propose the following Markov Chain Monte Carlo (MCMC) method to draw samples from the posterior density function; and in turn compute the Bayes estimates, and also construct HPD credible intervals.

\section{B. Gibbs Sampling}

For generating samples from the posterior density function (29), we assume that the PDF of $\pi_{1}(\lambda \mid a, b)$ follows $\operatorname{Gamma}(a, b)$, and the PDF of $\pi_{2}(\alpha \mid c, d)$ follows Gamma $(c, d)$. We will observe later that our result holds for a much more general class of distributions of $\pi_{2}(\alpha \mid c, d)$ rather than just the gamma distribution. We need the following results for further developments.

Theorem 1: Conditionally on $\alpha$, and the data, $\lambda$ follows a gamma distribution with the shape parameter $D+a$, and the scale parameter $\left.\sum_{i=1}^{D} y_{i: n}^{\alpha}+(n-D) U^{\alpha}+b\right)$; that is $\operatorname{Gamma}\left(D+a, \sum_{i=1}^{D} y_{i: n}^{\alpha}+(n-D) U^{\alpha}+b\right)$.

The proof is straight forward, and therefore omitted.

Theorem 2: The conditional distribution of $\alpha$ given the data is log-concave.

Proof: See the Appendix, part B.

Devroye [7] proposed a general method to generate samples from a general log-concave density function. Therefore, using Theorems 1 and 2, and adopting the method of Devroye [7], it is possible to generate samples from (29). We use the idea of Geman \& Geman [13] to generate samples from the conditional posterior density function using the following scheme.

Step 1) Generate $\alpha_{1}$, from the posterior density function $l(\cdot \mid$ data $)$, as given in (35), using the method proposed by Devroye [7].

Step 2) Generate $\lambda$ from $\pi_{1}\left(\cdot \mid \alpha_{1}\right.$, data $)$ as provided in Theorem 1.

Step 3) Repeat Steps 1 and 2, $M$ times, and obtain $\alpha_{i}$, and $\lambda_{i}$ for $i=1, \ldots, M$.

Step 4) Bayes estimates of $\alpha$, and $\lambda$ with respect to a squared error loss function can be obtained as

$$
\widehat{\alpha}_{\text {Bayes }}=\frac{1}{M} \sum_{i=1}^{M} \alpha_{i} \quad \text {, and } \quad \widehat{\lambda}_{\text {Bayes }}=\frac{1}{M} \sum_{i=1}^{M} \lambda_{i}
$$

respectively.

Step 5) If $\alpha_{(1)}<\ldots<\alpha_{(M)}$ are the ordered $\alpha_{i}$, and suppose we want to construct a $100(1-\beta) \%$ HPD credible interval of $\alpha$, then consider the following $100(1-\beta) \%$ credible intervals of $\alpha$ :

$$
\left(\alpha_{(1)}, \quad \alpha_{((1-\beta) M)}\right), \quad \ldots \quad,\left(\alpha_{(\beta M)}, \quad \alpha_{(M)}\right),
$$

Choose that interval which has the shortest length. Similarly, we can construct a $100(1-\beta) \%$ HPD credible interval of $\beta$.

\section{NUMERICAL EXPERIMENTS}

In this section, we present some experimental results, mainly to observe how the different methods behave for different sample sizes, and for different censoring schemes. We estimate the unknown parameters using the MLE, AMLE, Bayes estimators obtained by Lindley's approximations, and also by the Bayes estimators obtained by using MCMC technique. We compare the performances of the different estimators with respect to their average biases, and MSE. 
We also compare the average lengths of the asymptotic confidence intervals to the HPD credible intervals by their coverage percentages. From now on, the Bayes estimators obtained by Lindley's approximation, and by using the MCMC technique, will be denoted by BEL, and BEM respectively.

All the experiments have been performed at the Indian Institute of Technology Kanpur on a Pentium IV processor using FORTRAN-77. We have used the random deviate generator RAN2 of Press et al. [23]. Because $\lambda$ is the scale parameter, we have taken $\lambda=1$ without loss of generality. Moreover, we present the result when $T$ is of the form $T^{(1 / \alpha)}$, because in that case if $\widehat{\alpha}$ represents the MLE or AMLE of $\alpha$, then the distribution of $(\hat{\alpha} / \alpha)$ is independent of $\alpha$. Because of that result, we report the result only for $\alpha=1$; but these results can be used for any other $\alpha$, as we will explain later.

For computing the Bayes estimators, we have assumed that $\alpha$, and $\lambda$ have respectively $\operatorname{Gamma}(a, b)$, and $\operatorname{Gamma}(c, d)$ priors. To make the comparison meaningful, it is assumed that the priors are non-informative, and they are $a=b=c=d=$ 0 . Note that in this case the priors are non-proper also. When $c=d=0, \pi_{2}(\alpha)$ is not log-concave, but the posterior density function $\pi_{2}(\alpha \mid$ data $)$ is still log-concave.

Press [22] suggested to use very small non-negative values of the hyper parameters in this case, and it will make the priors proper. We have tried $a=b=c=d=0.0001$. The results are not significantly different than the corresponding results obtained using non-proper priors, and are not reported due to space.

We have considered different $n, R$, and $T$ values. In each case, we compute MLE, AMLE, BEL, and BEM. We compute the average estimates, and MSE over 1000 replications. We have also computed approximate $95 \%$ confidence intervals of the unknown parameters using the asymptotic distribution of the MLE, and also the HPD credible intervals using Gibbs samples. We replicate the process 1000 times; and report the average confidence/credible lengths, and coverage percentages. All the results are reported in Tables I-IV.

Some of the points are quite clear from the experiments. For all the methods, and for both the estimators, it is observed that for fixed $n$ as $R$ increases, or $T$ increases the average biases, and the MSE decrease. The average confidence intervals and credible lengths also decrease as $R$ or $T$ increases for fixed $n$. The AMLE work quite well in all the cases considered. Interestingly, the average biases of the AMLE are slightly smaller than the MLE, but the MSE are slightly larger, mainly for the scale parameters. The performance of the BEL are quite similar to the MLE in terms of biases, and MSE.

Now, considering the confidence intervals and credible lengths, it is observed that the asymptotic results of the MLE work quite well, even when the sample size is 30 . It can maintain the coverage percentages in most of the cases. However, when $R$ is small, and for $T=1$, the coverage percentages are significantly higher than the corresponding nominal level. But when we replace the MLE by AMLE, the results are not that satisfactory. In most of the cases, the coverage percentages are smaller than the nominal level.

The performance of the credible intervals of BEM are quite satisfactory. The coverage percentages are close to the nom-
TABLE I

The Average Biases, AND the MEAN SQuared ERrors of MLE, AMLE, BEL, AND BEM FOR $n=30, T=1.0 / T=2.0$

\begin{tabular}{|cc|c|c|c|c|}
\hline & & $R=22$ & $R=24$ & $R=26$ & $R=28$ \\
\hline \multirow{2}{*}{ MLE } & $T=1$ & $1.081(0.056)$ & $1.071(0.048)$ & $1.062(0.039)$ & $1.056(0.034)$ \\
& & $1.033(0.047)$ & $1.007(0.042)$ & $1.004(0.040)$ & $1.004(0.038)$ \\
& $T=2$ & $1.044(0.034)$ & $1.051(0.036)$ & $1.057(0.035)$ & $1.057(0.034)$ \\
& & $1.022(0.042)$ & $1.013(0.039)$ & $1.004(0.039)$ & $1.003(0.039)$ \\
\hline \multirow{2}{*}{ AMLE } & $T=1$ & $1.073(0.054)$ & $1.064(0.046)$ & $1.056(0.038)$ & $1.051(0.033)$ \\
& & $0.986(0.052)$ & $0.994(0.048)$ & $0.999(0.046)$ & $1.001(0.045)$ \\
& & & & & \\
& & & & & \\
BEL & $T=1$ & $1.038(0.034)$ & $1.045(0.035)$ & $1.051(0.034)$ & $1.052(0.034)$ \\
& & $1.079(0.056)$ & $1.005(0.044)$ & $1.001(0.044)$ & $1.001(0.045)$ \\
& $T=2$ & $1.042(0.034)$ & $1.049(0.036)$ & $1.056(0.036)$ & $1.057(0.036)$ \\
& & $1.016(0.040)$ & $1.009(0.039)$ & $0.998(0.037)$ & $0.999(0.037)$ \\
\hline \multirow{2}{*}{ BEM } & $T=1$ & $1.040(0.040)$ & $1.043(0.042)$ & $1.040(0.035)$ & $1.024(0.027)$ \\
& & $1.043(0.058)$ & $1.034(0.055)$ & $1.020(0.050)$ & $1.027(0.047)$ \\
& $T=2$ & $1.022(0.032)$ & $1.021(0.032)$ & $1.029(0.030)$ & $1.041(0.030)$ \\
& & $1.015(0.047)$ & $1.015(0.044)$ & $1.032(0.048)$ & $1.017(0.045)$ \\
\hline
\end{tabular}

TABLE II

The Average Biases, AND the MeAn SQuared ERrors of MLE, AMLE, BEL, AND BEM FOR $n=40, T=1.0 / T=2.0$

\begin{tabular}{|cc|c|c|c|c|}
\hline & & $R=28$ & $R=32$ & $R=36$ & $R=38$ \\
\hline \multirow{2}{*}{ MLE } & $T=1$ & $1.058(0.037)$ & $1.050(0.030)$ & $1.044(0.025)$ & $1.041(0.022)$ \\
& & $1.061(0.034)$ & $1.006(0.032)$ & $1.004(0.029)$ & $1.003(0.028)$ \\
& $T=2$ & $1.029(0.024)$ & $1.035(0.024)$ & $1.043(0.024)$ & $1.041(0.022)$ \\
& & $1.025(0.033)$ & $1.016(0.030)$ & $1.004(0.029)$ & $1.003(0.028)$ \\
\hline \multirow{2}{*}{ AMLE } & $T=1$ & $1.052(0.036)$ & $1.045(0.030)$ & $1.039(0.024)$ & $1.037(0.022)$ \\
& & $0.993(0.041)$ & $0.999(0.037)$ & $0.002(0.035)$ & $1.003(0.034)$ \\
& $T=2$ & $1.025(0.023)$ & $1.030(0.023)$ & $1.038(0.024)$ & $1.037(0.022)$ \\
& & $1.013(0.035)$ & $1.008(0.035)$ & $1.003(0.034)$ & $1.003(0.034)$ \\
\hline \multirow{2}{*}{ BEL } & $T=1$ & $1.056(0.037)$ & $1.049(0.031)$ & $1.043(0.026)$ & $1.041(0.024)$ \\
& & $1.057(0.033)$ & $1.003(0.032)$ & $1.000(0.028)$ & $1.000(0.027)$ \\
& $T=2$ & $1.028(0.024)$ & $1.033(0.024)$ & $1.042(0.025)$ & $1.041(0.024)$ \\
& & $1.022(0.033)$ & $1.013(0.030)$ & $1.000(0.028)$ & $1.000(0.027)$ \\
\hline \multirow{2}{*}{ BEM } & $T=1$ & $1.030(0.030)$ & $1.031(0.027)$ & $1.029(0.022)$ & $1.027(0.020)$ \\
& & $1.034(0.042)$ & $1.024(0.041)$ & $1.028(0.034)$ & $1.010(0.029)$ \\
& $T=2$ & $1.010(0.023)$ & $1.020(0.024)$ & $1.025(0.022)$ & $1.024(0.021)$ \\
& & $1.018(0.033)$ & $1.004(0.030)$ & $1.011(0.030)$ & $1.012(0.030)$ \\
\hline
\end{tabular}

inal level in most of the cases. Moreover, in most of the cases, the average lengths of the credible intervals are slightly shorter than the confidence intervals. Finally, note that Bayes estimates are most computationally expensive, followed by MLE, and AMLE.

Now we explain how we can use the table values for any other $\alpha$. For example, when $\alpha=2$, then for $n=30, R=22$, and $T=1$ (Table I), the average MLE of $\alpha$ will be $2 \times 1.081$, the MSE will be $4 \times 0.056$, the average $95 \%$ confidence length (Table III) will be $2 \times 0.7464$, and the coverage percentage will be $95 \%$. Similarly, when $\alpha=2$, for $n=30, R=22$, and $T=2^{(1 / 2)}$, the average MLE will be $2 \times 1.044$, the MSE will be $4 \times 0.034$, the average $95 \%$ confidence length (Table III) will be $2 \times 0.6490$, and the coverage percentage will be $95 \%$. 
TABLE III

THE AVERAGE 95\% CONFIDENCE INTERVALS OF THE MLE, AMLE, AND CREDible LengthS of BEM; AND THEIR Coverage PERCENTAGES FOR $n=30, T=1 . / T=2.0$

\begin{tabular}{|c|c|c|c|c|}
\hline & $R=22$ & $R=24$ & $R=26$ & $R=28$ \\
\hline \multirow{3}{*}{ MLE } & $0.7464(95)$ & $0.6999(95)$ & $0.6624(95)$ & $0.6262(95)$ \\
\hline & $0.8806(98)$ & $0.8018(93)$ & $0.7932(92)$ & $0.7857(92)$ \\
\hline & $0.6490(95)$ & $0.6464(95)$ & $0.6470(95)$ & $0.6227(95)$ \\
\hline & $0.8052(93)$ & $0.7846(93)$ & $0.7900(93)$ & $0.7815(92)$ \\
\hline \multirow{4}{*}{ AMLE } & $0.6676(92)$ & $0.6251(91)$ & $0.6200(92)$ & $0.5594(89)$ \\
\hline & $0.7906(89)$ & $0.7968(90)$ & $0.8450(90)$ & $0.7913(87)$ \\
\hline & $0.5791(93)$ & $0.5851(92)$ & $0.6056(92)$ & $0.5575(89)$ \\
\hline & $0.7812(92)$ & $0.7927(91)$ & $0.8435(90)$ & $0.7897(87)$ \\
\hline \multirow{4}{*}{ BEM } & $0.7665(97)$ & $0.6997(95)$ & $0.6564(95)$ & $0.6093(96)$ \\
\hline & $0.8491(95)$ & $0.7948(95)$ & 0.7601 (95) & $0.7498(96)$ \\
\hline & $0.6443(95)$ & $0.6425(95)$ & $0.6392(96)$ & $0.6105(95)$ \\
\hline & $0.7443(95)$ & $0.7500(95)$ & $0.7457(95)$ & $0.7313(94)$ \\
\hline
\end{tabular}

TABLE IV

THE AVERAGE 95\% CONFIDENCE INTERVALS OF THE MLE, AMLE, AND THE CRedible Lengths of BEM; AND Their Coverage Percentages for $n=40, T=1.0 / T=2.0$

\begin{tabular}{|c|c|c|c|c|}
\hline & $R=28$ & $R=32$ & $R=36$ & $R=38$ \\
\hline \multirow{4}{*}{ MLE } & $0.6506(96)$ & $0.6075(96)$ & $0.5509(95)$ & $0.5329(96)$ \\
\hline & 0.7969 (99) & $0.7042(94)$ & $0.6955(94)$ & $0.6923(94)$ \\
\hline & $0.5541(95)$ & $0.5618(96)$ & $0.5466(95)$ & $0.5329(96)$ \\
\hline & $0.6979(95)$ & $0.6977(95)$ & $0.6926(94)$ & $0.6912(94)$ \\
\hline \multirow{4}{*}{ AMLE } & $0.6011(94)$ & $0.5457(94)$ & $0.4970(93)$ & $0.4864(91)$ \\
\hline & 0.7289 (93) & $0.6922(93)$ & $0.6869(92)$ & $0.6865(90)$ \\
\hline & $0.4977(94)$ & $0.4985(94)$ & $0.4933(93)$ & $0.4864(91)$ \\
\hline & $0.6876(94)$ & $0.6864(93)$ & $0.6854(92)$ & $0.6849(90)$ \\
\hline \multirow{4}{*}{ BEM } & $0.6644(96)$ & $0.5983(95)$ & $0.5411(95)$ & 0.5133 (95) \\
\hline & $0.7495(96)$ & $0.6672(94)$ & $0.6466(95)$ & $0.6315(95)$ \\
\hline & $0.5457(94)$ & $0.5527(95)$ & $0.5315(95)$ & $0.5043(94)$ \\
\hline & $0.6408(94)$ & $0.6400(95)$ & $0.6315(95)$ & $0.6310(94)$ \\
\hline
\end{tabular}

\section{DATA ANALYSis}

In this section, we present one data analysis for illustrative purposes. It is strength data originally reported by Badar \& Priest [2]. The authors are thankful to Professor R.G. Surles for providing the data, which represent the strength measured in GPA for single carbon fibers, and impregnated 1000-carbon fiber tows. Single fibers were tested under gauge lengths of 1 , 10,20 , and $50 \mathrm{~mm}$. Impregnated tows of 1000 fibers were tested at gauge lengths of $20,50,150$, and $300 \mathrm{~mm}$. We will be considering the single fibers of $10 \mathrm{~mm}$ gauge length, with sample size $n=63$. The data are available in Kundu \& Gupta [19]. We use the data after subtracting 1.75, and consider the following two sampling schemes:

Scheme 1 is $R=40, \quad$ and $T=2.0$; and Scheme 2 is $R=20$, and $T=1.0$.

Before progressing, first we want to check whether the Weibull distribution fits the data or not, and that we have used the complete data. In this case, the MLE, AMLE, BEL, and

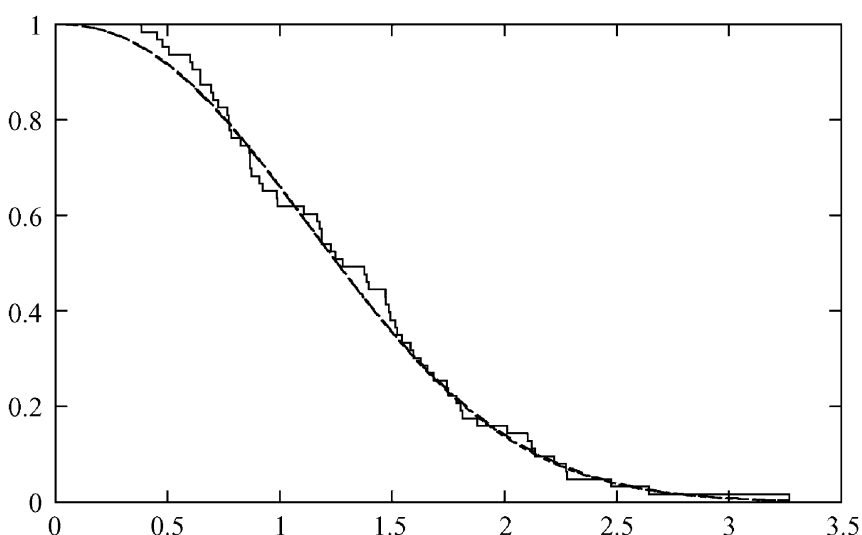

Fig. 2. Empirical, and fitted survival functions for the complete data set.

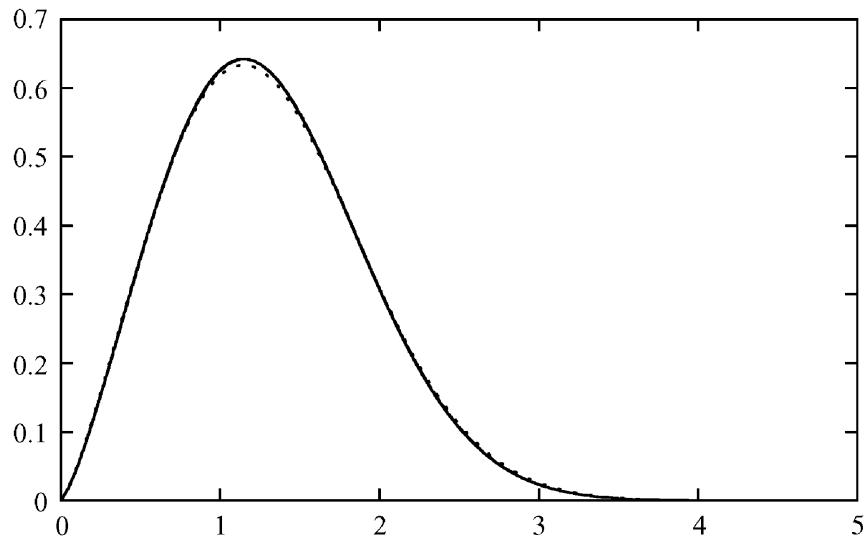

Fig. 3. Different estimated density functions for the scheme 1 data set.

BEM of the unknown parameters $(\alpha, \lambda)$ are $(2.2560,0.4130)$, (2.2611, 0.4152), (2.2498, 0.4165), and (2.2300, 0.4176) respectively. The corresponding Kolmogorov-Smirnov statistics, and the associated $p$ values (in brackets) are $0.0723(0.8967)$ 0.0711(0.9076), 0.0701(0.9160), and 0.0691(0.9244) respectively. We have just plotted the empirical survival function, and the fitted survival functions in Fig. 2. Observe that all the four fitted survival functions are almost identical, and they fit the data very well.

Now for scheme 1, the MLE, AMLE, BEL, and BEM of $(\alpha, \lambda)$ are $(2.2985,0.4099),(2.2935,0.4122),(2.2945,0.4125)$, and $(2.2680,0.4120)$ respectively. The $95 \%$ confidence intervals based on MLE, AMLE, and the credible lengths based on BEM of $(\alpha, \lambda)$ are $\{(1.7838,2.8131),(0.2640,0.5559)\}$, $\{(1.7794,2.8076),(0.2654,0.5590)\}$, and $\{(1,7860,2.8133)$, $(0.2847,0.5804)\}$ respectively. We have plotted the different estimated density functions in Fig. 3; observe that they are almost identical.

For scheme 2, the MLE, AMLE, BEL, and BEM of $(\alpha, \lambda)$ are $(2.8544,0.5072),(2.8446,0.5077),(2.8404,0.5067)$, and $(2.7894,0.4987)$ respectively. The $95 \%$ confidence intervals based on MLE, AMLE, and the credible lengths based on BEM of $(\alpha, \lambda)$ are $\{(1.7980,3.9108)(0.3067,0.7077)\}$, $\{(1.7917,3.8974),(0.3070,0.7084)\}$, and $\{(1.8436,3.9430)$, $(0.3258,0.7263)\}$ respectively. We plot all the estimated density functions in Fig. 4. Observe that the density function 


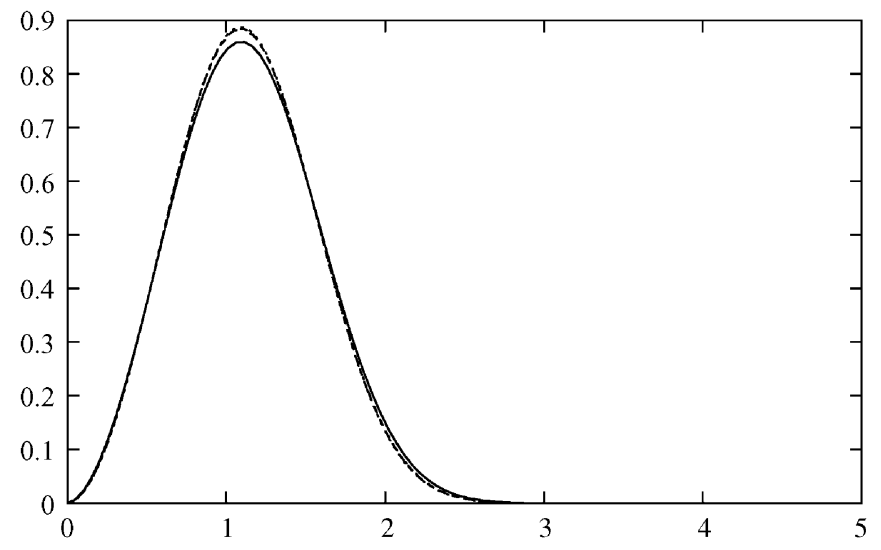

Fig. 4. Different estimated density functions for the scheme 2 data set.

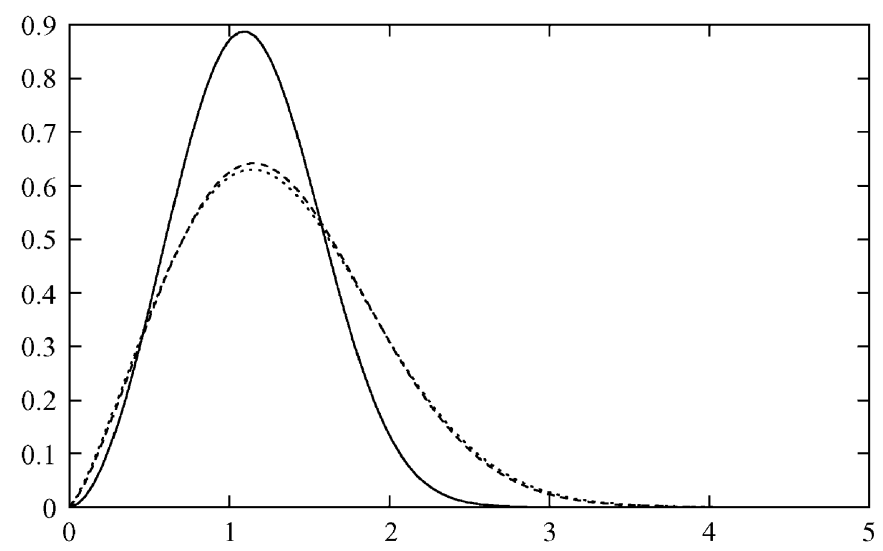

Fig. 5. Different estimated density functions based on MLE for complete, scheme 1 , and scheme 2 data sets.

obtained using BEM is slightly different than the other three density functions.

Now, we want to see the effect of censoring on the estimation of the unknown parameters. We have plotted the three density functions based on MLE for complete, scheme 1, and scheme 2 data sets in Fig. 5. Observe that the estimated density functions for complete, and scheme 1 data sets are almost identical. For scheme 1 , the effect is not noticeable; but for scheme 2 , the effect is quite severe. It is clear that the estimated density function obtained using the scheme 2 data set could not estimate the right tail properly because of the absence of information in that region.

\section{OPTIMUM Censoring SCHEME}

In practice, it is quite important to choose the optimum censoring scheme among possible different schemes. In this section, we briefly describe how to choose an optimum censoring scheme among possible schemes. Here, a possible Type-II hybrid censoring scheme means, for a fixed sample size $n$, a particular choice of $(T, R)$, when $R \leq n$. Therefore, a practitioner might be interested in choosing that particular Type-II hybrid censoring scheme, which provides the maximum information of the unknown parameters. Another important problem might be to choose between a Type-I, and Type-II hybrid censoring scheme. For example, if there is a Type-I hybrid censoring scheme $\left[T_{1}, R_{1}\right]$, and a Type-II hybrid censoring scheme
$\left(T_{2}, R_{2}\right)$, one is interested to know which one provides more information about the unknown parameters. In both the problems, one of the common points is to define an information measure for a given censoring scheme. Note that, if only one parameter is unknown, then it can be defined as a measure which is inversely proportional to the asymptotic variance of the unknown parameter; see Zhang \& Meeker [24]. But if both the parameters are unknown, then it is not very easy to define. Some of the existing choices are the trace, or determinant of the Fisher information matrix. But unfortunately, they are not scale invariant; see Gupta \& Kundu [15].

One way to define the information measure for a particular sampling scheme is as the inverse of the asymptotic variance of the $100 p$-th quantile estimator obtained using that particular scheme; see for example Zhang \& Meeker [24]. Because this information measure depends on $p$, recently Gupta \& Kundu [15] proposed the information measure

$$
I\{(T, R)\}=\left[\int_{0}^{1} V\left((T, R)_{p}\right) d p\right]^{-1},
$$

where $V\left((T, R)_{p}\right)$ denotes the asymptotic variance of the 100 $p$-th quantile estimator obtained using the Type-II hybrid censoring scheme $(T, R)$; or a more general information measure can be defined as

$$
I^{*}\{(T, R)\}=\left[\int_{0}^{1} w(p) V\left((T, R)_{p}\right) d p\right]^{-1} .
$$

Here, $w(p)$ is a non-negative weight function defined on $(0$, 1). Note that the information measure proposed by Zhang \& Meeker [24] is a particular case of (32). Similarly, we can define $I\{[T, R]\}$ or $I^{*}\{[T, R]\}$ also.

Now, among the different Type-II hybrid censoring schemes, if somebody wants to choose that scheme which provides the maximum (31) or (32), the obvious choice should be $R=n$. Similarly, between a Type-I hybrid censoring scheme $\left[T_{1}, R_{1}\right]$, and a Type-II hybrid censoring scheme $\left(T_{2}, R_{2}\right)$, one should prefer that particular scheme which provides the maximum information measure. Moreover, between the Type-I hybrid censoring scheme $[T, R]$, and Type-II hybrid censoring scheme $(T, R)$, clearly $(T, R)$ provides more information than $[T, R]$. Therefore, without restricting the duration of the experiment, our result is not important. One way to bring the time into consideration is to introduce a cost factor on time. In this paper, we do not use the cost factor approach. Instead we choose that scheme which provides the maximum information measure among the schemes with a given maximum expected duration of the experiment.

To choose the optimum censoring scheme, or to choose between a Type-I or a Type-II hybrid censoring scheme, the experimenter needs to know the unknown parameters. In practice, if it is completely unknown, the experimenter needs to perform some pilot experiments to approximate the unknown parameters. We observed in the numerical study, although we could not 
prove it theoretically, that for fixed $n$ and $T$ as $R$ increases, or for fixed $n$ and $R$ as $T$ increases, the information measure (31) increases. Therefore, for the Type-II hybrid censoring scheme for the given time period $T^{0}$, we choose the maximum $R$, say $R^{*}$, such that $E\left(Y_{R^{*}: n}\right) \leq T^{0}$. This result means that among all the possible Type-II hybrid censoring schemes, whose expected durations are less than $T^{0},\left(T^{0}, R^{*}\right)$ has the maximum $I\{(R, T)\}$ value. Similarly, for the Type-I hybrid censoring case also, for fixed $n$ and $T$ as $R$ increases, or for fixed $n$ and $R$ as $T$ increases, the information measure $I\{[T, R]\}$ increases. Therefore, for a Type-I hybrid censoring scheme, among all the possible Type-I hybrid censoring schemes whose expected durations are less than $T^{0},\left[T^{0}, R^{*}+1\right]$ has the maximum $I\{[T, R]\}$ value. Similarly, for fixed $T^{0}$, the choice between $\left(T^{0}, R^{*}\right)$, and $\left[T^{0}, R^{*}+1\right]$ can be easily made based on their information measures.

Now we discuss with an illustrative example how to choose the optimum scheme for fixed $n$; and when the shape, and scale parameters are known. Because $\lambda$ is the scale parameter, we take $\lambda=1$, without loss of generality. We illustrate the result for $\alpha=2.0$, and $n=10$. Now, if we put 10 items on test, then without any censoring mechanism, the expected duration of the experiment is $E\left(Z_{(10)}\right)$, where $Z_{(10)}$ is the maximum order statistic of a sample of size 10 , from a Weibull distribution with the shape parameter 2 , and scale parameter 1 . Therefore,

$$
E\left(Z_{(10)}\right)=20 \int_{0}^{\infty} x^{2} e^{-x^{2}}\left(1-e^{-x^{2}}\right)^{9} d x=1.6757 \approx 1.68
$$

indicates that, without any censoring mechanism, the expected duration of the experiment is 1.68 units of time. But suppose the experimenter can afford only 1.26 units $(\approx 75 \%$ of 1.68$)$ of time. Then what should be the optimum choice? Observe that $E\left(Z_{(8)}\right)<1.26<E\left(Z_{(9)}\right)$. Therefore, the optimum Type-I, and Type-II hybrid censoring sampling schemes will be $\{[1.26$, $9]\}$, and $\{(1.26,8)\}$ respectively; and between the two options, choose the one which produces the higher information measure.

\section{CONCLUSIONS}

In this paper, we consider the classical, and Bayesian inference procedures for the Type-II hybrid censored Weibull parameters. We provide the maximum likelihood estimators, and it is observed that the maximum likelihood estimators of the unknown parameters can be obtained by solving a simple iterative procedure. We have also proposed approximate maximum likelihood estimators of the unknown parameters, and they can be obtained explicitly. The Bayes estimators of the unknown parameters can not be obtained in explicit forms, and we have proposed two approximations which can be implemented very easily. We compare the performance of the different methods by Monte Carlo simulations, and it is observed that the performances are quite satisfactory.

\section{APPENDIX A}

For the two-parameter case, using notation $\left(\lambda_{1}, \lambda_{2}\right)=(\alpha, \lambda)$, the Lindley's approximation can be written as

$$
\begin{array}{r}
\widehat{g}=g\left(\widehat{\lambda}_{1}, \widehat{\lambda}_{2}\right)+\frac{1}{2}\left(A+l_{30} B_{12}+l_{03} B_{21}+l_{21} C_{12}+l_{12} C_{21}\right) \\
+p_{1} A_{12}+p_{2} A_{21},
\end{array}
$$

where

$$
\begin{aligned}
A= & \sum_{i=1}^{2} \sum_{j=1}^{2} w_{i j} \tau_{i j}, l_{i j}=\frac{\partial^{i+j} L\left(\lambda_{1}, \lambda_{2}\right)}{\partial \lambda_{1}^{i} \partial \lambda_{2}^{j}}, \\
& i, j=0,1,2,3, \quad \& \quad i+j=3, \\
p_{i}= & \frac{\partial p}{\partial \lambda_{i}}, w_{i}=\frac{\partial g}{\partial \lambda_{i}}, w_{i j}=\frac{\partial^{2} g}{\partial \lambda_{i} \partial \lambda_{j}}, p=\ln \pi\left(\lambda_{1}, \lambda_{2}\right), \\
A_{i j}= & w_{i} \tau_{i i}+w_{j} \tau_{j i}, B_{i j}=\left(w_{i} \tau_{i i}+w_{j} \tau_{i j}\right) \tau_{i i}, \\
c_{i j}= & 3 w_{i} \tau_{i i} \tau_{i j}+w_{j}\left(\tau_{i i} \tau_{j j}+2 \tau_{i j}^{2}\right) .
\end{aligned}
$$

Now,

$$
\begin{aligned}
L(\alpha, \lambda)= & D \ln \alpha+D \ln \lambda(\alpha-1) \sum_{i=1}^{D} \ln y_{i: n} \\
& -\lambda\left\{\sum_{i=1}^{D} y_{i: n}^{\alpha}+(n-D) U^{\alpha}\right\}, \\
l_{30}= & \frac{2 D}{\widehat{\alpha}^{3}}-\widehat{\lambda}\left[\sum_{i=1}^{D} y_{i: n}^{\alpha}\left(\ln y_{i: n}\right)^{3}+(n-D) U^{\alpha}(\ln U)^{3}\right], \\
l_{03}= & \frac{2 D}{\widehat{\lambda}^{3}}, \\
l_{21}= & -\left[\sum_{i=1}^{D} y_{i: n}^{\widehat{\alpha}}\left(\ln y_{i: n}\right)^{2}+(n-D) U^{\alpha}(\ln U)^{2}\right], \\
l_{12}= & 0 .
\end{aligned}
$$

Now, when $g(\alpha, \lambda)=\alpha$, then

$$
w_{1}=1, \quad w_{2}=0, \quad w_{i j}=0, \quad \text { for } \quad i, j=1,2 .
$$

Therefore,

$$
\begin{aligned}
A & =0, \quad B_{12}=\tau_{11}^{2}, \quad B_{21}=\tau_{21} \tau_{22}, \quad C_{12}=3 \tau_{11} \tau_{12}, \\
C_{21} & =\left(\tau_{22} \tau_{11}+2 \tau_{21}^{2}\right), \quad A_{12}=\tau_{11} \quad A_{21}=\tau_{12} . \\
p & =\ln \pi_{2}(\alpha)+\ln \pi_{1}(\lambda) \\
& =(a-1) \ln \lambda-b \lambda+(c-1) \ln \alpha-d \alpha, \quad \text { and } \\
p_{1} & =\frac{c-1}{\widehat{\alpha}}-d, \quad p_{2}=\frac{a-1}{\widehat{\lambda}}-b .
\end{aligned}
$$

Now for the second part, when $g(\alpha, \lambda)=\lambda$, then

$$
\begin{aligned}
w_{1} & =0, \quad w_{2}=1, \quad w_{i j}=0 \quad \text { for } \quad i, j=1,2, \quad \text { and } \\
A & =0, \quad B_{12}=\tau_{12} \tau_{11}, \quad B_{21}=\tau_{22}^{2}, \\
C_{12} & =\tau_{11} \tau_{22}+2 \tau_{12}^{2}, \\
C_{21} & =3 \tau_{22} \tau_{21}, \quad A_{12}=\tau_{21}, \quad A_{21}=\tau_{22} .
\end{aligned}
$$




\section{APPENDIX B}

The conditional density of $\alpha$, and $\lambda$ given the data is

$$
\begin{aligned}
l(\alpha, \lambda \mid \text { data }) \propto \pi_{2}(\alpha) & \alpha^{D} \lambda^{D+a-1} \\
& \times \prod_{i=1}^{D} y_{i: n}^{\alpha-1} e^{-\lambda\left(\sum_{i=1}^{D} y_{i: n}^{\alpha}+(n-D) U^{\alpha}+b\right) .}
\end{aligned}
$$

The conditional density of $\alpha$ given the data is

$$
l(\alpha \mid \text { data }) \propto \frac{\pi_{2}(\alpha) \alpha^{D} \prod_{i=1}^{D} y_{i: n}^{\alpha}}{\left(\sum_{i=1}^{D} y_{i: n}^{\alpha}+(n-D) U^{\alpha}+b\right)^{a+D}}
$$

The log-likelihood of $l(\alpha \mid$ data $)$ is

$$
\begin{aligned}
\ln l(\alpha \mid \text { data }) & =\ln \pi_{2}(\alpha)+D \ln \alpha+\alpha \sum_{i=1}^{D} \ln y_{i: n} \\
& -(a+D) \ln \left(\sum_{i=1}^{D} y_{i: n}^{\alpha}+(n-D) U^{\alpha}+b\right)
\end{aligned}
$$

Because $\pi_{2}(\alpha)$ is log-concave, and using Lemma 1 of Kundu [18], it follows that

$$
\frac{d}{d \alpha^{2}} \ln \left(\sum_{i=1}^{D} y_{i: n}^{\alpha}+(n-D) U^{\alpha}+b\right) \geq 0 .
$$

Therefore, the result follows.

\section{REFERENCES}

[1] B. Arnold, B. N. Balakrishnan, and H. N. Nagaraja, A First Course in Order Statistics. New York: Wiley, 1992.

[2] M. G. Badar and A. M. Priest, "Statistical aspects of fiber and bundle strength in hybrids composites," in Progress in Science and Engineering Composites, T. Hayashi, K. Kawata, and S. Umekawa, Eds. Tokyo: , 1982, pp. 1129-1136, ICCM-IV.

[3] U. Balasooriya and N. Balakrishnan, "Reliability sampling plans for log-normal distribution, based on progressively-censored samples," IEEE Trans. Reliability, vol. 49, pp. 199-203, 2000.

[4] J. O. Berger and D. Sun, "Bayesian analysis for the Poly-Weibull distribution," Journal of the American Statistical Association, vol. 88, pp. 1412-1418, 1993.

[5] S. Chen and G. K. Bhattacharya, "Exact confidence bounds for an exponential parameter under hybrid censoring," Communications in Statistics-Theory and Methods, vol. 17, pp. 1857-1870, 1988.

[6] A. Childs, B. Chandrasekhar, N. Balakrishnan, and D. Kundu, "Exact likelihood inference based on type-I and type-II hybrid censored samples from the exponential distribution," Annals of the Institute of Statistical Mathematics, vol. 55, pp. 319-330, 2003.

[7] L. Devroye, "A simple algorithm for generating random variates with a log-concave density," Computing, vol. 33, pp. 247-257, 1984.

[8] N. Draper and I. Guttman, "Bayesian analysis of hybrid life tests with exponential failure times," Annals of the Institute of Statistical Mathematics, vol. 39, pp. 219-225, 1987.

[9] N. Ebrahimi, "Estimating the parameter of an exponential distribution from hybrid life test," Journal of Statistical Planning and Inference, vol. 14, pp. 255-261, 1986.
[10] N. Ebrahimi, "Prediction intervals for future failures in exponential distribution under hybrid censoring," IEEE Trans. Reliability, vol. 41, pp. 127-132, 1992.

[11] B. Epstein, "Truncated life tests in the exponential case," Annals of Mathematical Statistics, vol. 25, pp. 555-564, 1954.

[12] K. Fairbanks, R. Madison, and R. Dykstra, "A confidence interval for an exponential parameter from a hybrid life test," Journal of the American Statistical Association, vol. 77, pp. 137-140, 1982.

[13] S. Geman and A. Geman, "Stochastic relaxation, Gibbs distributions and the Bayesian restoration of images," IEEE Trans. Pattern Analysis and Machine Intelligence, vol. 6, pp. 721-740, 1984.

[14] R. D. Gupta and D. Kundu, "Hybrid censoring schemes with exponential failure distribution," Communications in Statistics-Theory and Methods, vol. 27, pp. 3065-3083, 1988.

[15] R. D. Gupta and D. Kundu, "On the comparison of Fisher information matrices of the Weibull and generalized exponential distributions," Journal of Statistical Planning and Inference, vol. 136, pp. 3130-3144, 2006.

[16] H. S. Jeong, J. I. Park, and B. J. Yum, "Development of (r, T) hybrid sampling plans for exponential lifetime distributions," Journal of Applied Statistics, vol. 23, pp. 601-607, 1996.

[17] M. P. Kandinsky and V. V. Krivtsov, "A simple procedure for Bayesian estimation of the Weibull distribution," IEEE Trans. Reliability Analysis, vol. 54, no. 4, pp. 612-616, 2005.

[18] D. Kundu, "On hybrid censoring Weibull distribution," Journal of Statistical Planning and Inference, vol. 137, pp. 2127-2142, 2007.

[19] D. Kundu and R. D. Gupta, "Estimation of $P(Y<X)$ for Weibull distribution," IEEE Trans. Reliability, vol. 55, no. 2, pp. 270-280, 2006.

[20] D. V. Lindley, "Approximate Bayesian method," Trabajos de Estadistica, vol. 31, pp. 223-237, 1980

[21] Reliability Design Qualifications and Production Acceptance Test, Exponential Distribution, MIL-STD-781- C, U.S. Government Printing Office, Washington, DC, (1977).

[22] S. J. Press, The subjectivity of scientists and the Bayesian approach. New York: Wiley, 2001.

[23] W. H. Press, B. P. Flannery, S. A. Teukolsky, and W. T. Vetterling, Numerical Recipes: The Art of Scientific Computing. UK: Cambridge University Press, 1991.

[24] Y. Zhang and W. Q. Meeker, "Bayesian life test planning for the Weibull distribution with given shape parameter," Metrika, vol. 61, pp. 237-249, 2005.

Aveek Banerjee is a Business Analyst working in the consulting division of Accenture. He is presently working in the Customer Relationship Management (CRM) - Analytics domain of Accenture where he mainly develops statistical models on customer data, and provides the clients with the necessary solutions to their business problems. He holds an integrated MSc. degree in Mathematics and Scientific Computing from the Indian Institute of Technology (IIT) Kanpur. His research interests are in Survival Analysis, Statistical Modeling, Inference, and Data Mining.

Debasis Kundu is a Professor of Statistics in the Department of Mathematics and Statistics at the Indian Institute of Technology Kanpur. He received the B-Stat in 1982, and M.Stat in 1984 both from the Indian Statistical Institute, M.A (Mathematics) in 1985 from the University of Pittsburgh; and the Ph.D. in 1989 from the Pennsylvania State University. He has worked as an Assistant Professor at the University of Texas at Dallas from 1989 to 1990, and then joined Indian Institute of Technology Kanpur as an Assistant Professor in 1990. He became Professor in 1999. He is a Fellow of the National Academy of Sciences India. He is an Associate Editor of Communications in Statistics, Ser A and Ser B. His research interests include Statistical Signal Processing, Reliability Analysis, Statistical Computing, and Competing Risks Models. He has more than 125 publications in different peer reviewed journals. 